\title{
Sleep staging: Good old $R \&$ K! Do we need a revised AASM criteria?
}

\author{
J. M. Joshi \\ Dept. of Respiratory Medicine, B.Y.L. Nair Charitable Hospital, Mumbai
}

Indian J Sleep Med 2009; 4.1, 4-5

\section{History of sleep staging}

7

he stages of sleep were first described in 1937 by Alfred Lee Loomis and coworkers who separated different EEG features of sleep into 5 levels (A to $E$ ) representing the spectrum from wakefulness to deep sleep. In 1953, REM sleep was discovered as distinct phase and thusW illiam D ement and $N$ athaniel K leitman reclassified sleep into 4 N REM stages and REM . Thestaging criteria werestandardized in 1968 by Allan Rechtschaffen and Anthony Kales in the "R \& K sleep scoring system". [1] In the R\& K standard, normal sleep is divided into N REM and REM sleep. NREM sleep was divided into 4 stages, stage I (light sleep/theta wave), stage II (sleep spindles/ K complex), stages III and IV (deep sleep / slow-waveor deltawave), In stage 3, delta waves constutre less than $50 \%$ of the total wave-patterns, and more than $50 \%$ in stage 4 . Furthermore, REM sleep is sometimes referred to as stage 5. EffectiveJ uly 1, 2008, the American Academy of Sleep $M$ edicine (AASM) scoring manual replaces the "R\& $K$ Scoring Bible". [2] AASM mandated the use of its new scoring manual by AASM -accredited sleep centers. The rulesalso statethat new PSG equipment purchased on or after July 1, 2008, must fulfill the revised technical and digital specifications.

\section{American Academy of Sleep Medicine (AASM) sleep staging}

Amongst several changes, the most significant was the combination of stages 3 and 4 into StageN 3.T he sequence

Address for correspondence

\section{Dr. J. M. Joshi}

Professor and Head

Dept of Respiratory Medicine

B.Y.L. Nair Ch Hospital, Mumbai 400008

Tel/FaxN o: 91-022-23003095

Indian Journal of Sleep M edicine (IJSM ), Vol. 4, N o. 1, 2009 of N REM sleep stages 1 to 4 (R\& $K$ classification) arenow $N$ 1 to $N 3$ (AASM classification). Wakenow being referred to as stageW, REM should now belabeled stageR. Arousals respiratory, cardiac, and movement events werealso added [2]. Thenew AASM scoring manual proposestheaddition of frontal leads to the $R \& K$ manual specified central $E E G$ derivations (either $\mathrm{C} 3-\mathrm{A} 2$ or $\mathrm{C} 4-\mathrm{A} 1$ ) for the scoring of higher percentages of slow wave sleep. Frontal EEG leads are more likely to detect eye movements, which could be misinterpreted as slow EEG waves. Theoccurrence of frontal intermittent delta activity, a phenomenon sometimes associated with cognitiveimpairment in theelderly, may also be misinterpreted as slow wavesleep. M oreover, as stages 3 and 4 sleep are combined, the importance of quantifying deep slow wavesleep is diminished. Finally, the clinical value of elevating the overall percentage of slow wave sleep remains unclear. Thenew manual also offerstheoption of alternative EOG derivations, using Fpz as a reference for theright and left outer canthus electrodes to differentiate between vertical and horizontal eye movements, a distinction that has no practical value. U sing the alternativeEO $\mathrm{G}$ derivations eye movements that appear as in-phase deflections can easily be confused with artifacts. [3] T he continuous positiveairway pressure (CPAP) headgear used during titration sleep studies may increase the incidence of these artifacts by pressing against the Fpz electrode. H owever, asidefrom theaddition of frontal leadsand theoption of alternativederivationsmost proposed changes to the $R \& \mathrm{~K}$ manual are relatively minor. $\mathrm{N}$ omenclature changes include the renaming of stages 1,2 , 3 , and 4 sleep as N 1, N 2, and N 3, with stages 3 and 4 combined. Stage REM is renamed as stage R. The $A 1$ and $\mathrm{A} 2$ referenceelectrodesites are renamed as $\mathrm{M} 1$ and $\mathrm{M} 2$. A few modifications are made to mark the beginning and ending of sleep stages. The 3-minute rule for continuing stage 2 sleep in theabsence of K-complexes and sleep spindles has been discontinued, as well astheuse of "movement time" to denoteepochs that are unscorable due to artifact. [3] 


\section{Additional problems}

Visual sleep scoring is considered the "G old standard" for sleep analysis and scoring, necessary for sleep staging. Although visual sleep scoring was appropriate and sufficient for few signals recorded in the analog mode as curves on paper.[4] This staging may be insufficient for digitally recorded and stored multichannel sleep data and imposes a heavy load on the human visual analyzer.[4] Computer algorithms have been constructed to match visual scoring with the $R \& K$ rules as closely as possible, AASM will require new algorithms and the cost of changing the existing equipments is not justified [5].

\section{Summary}

The AASM manual is largely consensus-based and does not meet scientific evidence levels of standard practice parameters. The task force members involved in the consensus did not have an opportunity to review the contents of themanual beforeitspublication. Themanual presents a number of technical and practical concerns that remain unresolved Consensus-based recommendations are generally scrutinized, debated, and are eventually either accepted or rejected on the basis of merit and practicality. This was the case with the $\mathrm{R}$ and $\mathrm{K}$ manual nearly 40 years ago. It seems reasonable to expect the samelevel of scrutiny of the AASM manual, beforethe changes are implemented as a new standard. [3] R and $\mathrm{K}$ system was useful as it established minimal criteriafor staging sleep that efficiently produced comparable and relatively accurateresultsfor theclinician or researcher, among studies, scorers, and sleep laboratories. It allowed for different epoch sizes, but the 30-second epoch continued to provide adequatedetail supported by its continued, consistent use in clinical and research practice. Perhaps the old $\mathrm{R}$ and $\mathrm{K}$ scoring system was good enough!

\section{References}

1. Rechtschaffen A, Kales A. A Manual of Standardized Terminology, Techniques and Scoring Systems for Sleep Stages of Human Subjects. Los Angeles: UCLA Brain Information Services/Brain Research Institute; 1968.

2. Iber C. Ancoli-Israel S, Chesson A, Quan SF, for the American Academy of Sleep Medicine. The AASM Manual for the Scoring of Sleep and Associated Events: Rules, Terminology and Technical Specifications. Westchester, III: American Academy of Sleep Medicine; 2007.

3. Butkov N. Coming to consensus. Addressing practical and technical concerns with the new AASM Scoring Manual. Sleep Review. 2008;9:38-42.

4. Schulz H. Rethinking sleep analysis. J Clin Sleep Med 2008;4:99-103.

5. Butkov N. The Scoring Struggle. Sleep Review. Jan-Feb 2009. 